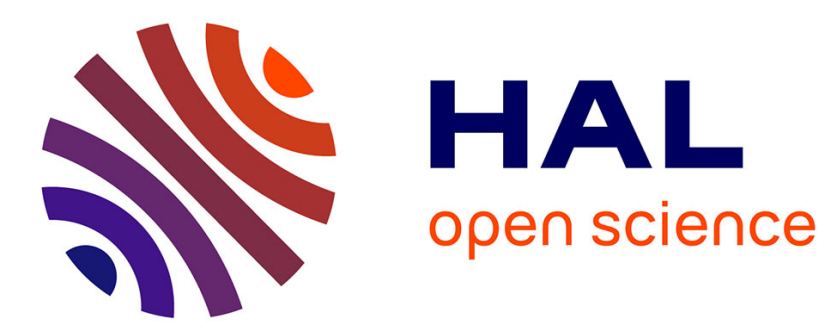

\title{
Unified Description of Saturation and Bistability of Intersubband Transitions in the Weak and Strong Light-Matter Coupling Regimes
}

\author{
Mathieu Jeannin, Jean-Michel Manceau, Raffaele Colombelli
}

\section{To cite this version:}

Mathieu Jeannin, Jean-Michel Manceau, Raffaele Colombelli. Unified Description of Saturation and Bistability of Intersubband Transitions in the Weak and Strong Light-Matter Coupling Regimes. Physical Review Letters, 2021, 127 (18), 10.1103/PhysRevLett.127.187401 . hal-03426810

\author{
HAL Id: hal-03426810 \\ https://hal.science/hal-03426810
}

Submitted on 12 Nov 2021

HAL is a multi-disciplinary open access archive for the deposit and dissemination of scientific research documents, whether they are published or not. The documents may come from teaching and research institutions in France or abroad, or from public or private research centers.
L'archive ouverte pluridisciplinaire HAL, est destinée au dépôt et à la diffusion de documents scientifiques de niveau recherche, publiés ou non, émanant des établissements d'enseignement et de recherche français ou étrangers, des laboratoires publics ou privés. 


\title{
Unified Description of Saturation and Bistability of Intersubband Transitions in the Weak and Strong Light-Matter Coupling Regimes
}

\author{
Mathieu Jeannin $\odot$, Jean-Michel Manceau๑, and Raffaele Colombelli॰* \\ Centre de Nanosciences et de Nanotechnologies (C2N), CNRS UMR 9001, Université Paris-Saclay, \\ 91120 Palaiseau, France
}

(Received 29 November 2020; revised 18 December 2020; accepted 20 September 2021; published 27 October 2021)

\begin{abstract}
We propose a unified description of intersubband absorption saturation for quantum wells inserted in a resonator, both in the weak and strong light-matter coupling regimes. We demonstrate how absorption saturation can be engineered. In particular, we show that the saturation intensity increases linearly with the doping in the strong coupling regime, while it remains doping independent in weak coupling. Hence, countering intuition, the most suitable region to exploit low saturation intensities is not the ultrastrong coupling regime, but is instead at the onset of the strong light-matter coupling. We further derive explicit conditions for the emergence of bistability. This Letter sets the path toward, as yet, nonexistent ultrafast midinfrared semiconductor saturable absorption mirrors (SESAMs) and bistable systems. As an example, we show how to design a midinfrared SESAM with a 3 orders of magnitude reduction in saturation intensity, down to $\approx 5 \mathrm{~kW} \mathrm{~cm}^{-2}$.
\end{abstract}

DOI: 10.1103/PhysRevLett.127.187401

Saturation of the light-matter interaction is a general feature of material systems, be they atoms or semiconductors [1]. In semiconductors, the possibility of judiciously controlling saturation phenomena is of great importance for fundamental physics as well as applications.

A seminal example is the development of the semiconductor saturable absorption mirror (SESAM) [2] based on interband transitions in semiconductor quantum wells (QWs) that revolutionized the field of ultrafast lasers in the visible and near-IR spectral range. Ultrafast lasers based on SESAMs now find applications in several domains, and even in quantum phenomena [3]. Saturable absorption can lead to optical bistability [1] that manifests itself as a hysteresis cycle in the input vs output characteristic of the system. Besides fundamental interests, it has been proposed as a resource for optical information processing. Proposals [4] and demonstrations [5] exist in the domain of exciton polaritons.

A saturable absorber exhibits an absorption coefficient $\alpha$ that depends on the incident intensity $I$ as

$$
\alpha=\frac{\alpha_{0}}{1+I / I_{\text {sat }}},
$$

where $\alpha_{0}$ is the absorption at low incident power, and $I_{\text {sat }}$ is the saturation intensity. At $I_{\text {sat }}$, the absorption is $50 \%$ of the low-intensity value. In general, $I_{\text {sat }}$ depends on the characteristics of the system: lifetimes, oscillator strengths, and operating frequency. The only way to engineer it is to act on the system lifetimes. Fortunately, the saturation intensities of systems based on interband transitions in semiconductors are naturally favorable for applications in the visible and near-IR spectral ranges.

The case is different for intersubband (ISB) transitions in QWs, the backbone of semiconductor-based mid-IR optoelectronics [quantum cascade lasers (QCLs), QW infrared detectors] [6,7]. Considering typical experimental configurations, the classical theory yields a saturation intensity in the absence of a cavity and/or resonator [8],

$$
I_{\mathrm{sat}}^{0}=\frac{\hbar^{2} \varepsilon_{0} c n_{\mathrm{opt}}}{2 e^{2} \tau_{12} T_{2}|\langle 1|z| 2\rangle|^{2}}=\frac{\varepsilon_{0} c n_{\mathrm{opt}}}{2 \tau_{12} T_{2}\left|\left(D_{12} / \hbar\right)\right|^{2}},
$$

where $n_{\mathrm{opt}} \approx 3.3$ is an average index of refraction of the active region, $\tau_{12} \approx 0.8 \mathrm{ps}$ is the upper state lifetime, $T_{2} \approx$ $60 \mathrm{fs}$ is the dephasing time, and $|\langle 1|e z| 2\rangle|=D_{12} \approx e \times$ $2 \mathrm{~nm}$ is the electric dipole matrix element. In the mid-IR $(\lambda=10 \mu \mathrm{m}), I_{\text {sat }}^{0} \approx 1 \mathrm{MW} / \mathrm{cm}^{2}$, as confirmed by a vast body of literature [9-11]. This very high value, that notably does not depend on the doping, explains why saturable absorbers, SESAM mirrors, and bistable systems are missing from the current toolbox of mid-IR optoelectronic devices: they could only be used with extremely high power laser sources and are incompatible with the typical output power levels of QCLs.

The nature of the absorption saturation for an ISB system is radically modified when operated in the strong lightmatter coupling regime. In this regime, new quasiparticles emerge (intersubband polaritons) with peculiar properties that motivate the recent flurry of activity. From their initial demonstration [12], a nonexhaustive list of current research includes electrically and optically pumped devices toward 
demonstrating bosonic final-state stimulation [13-17]. The ultrastrong coupling regime, where a sizable number of virtual photons is populating the ground state of the system, has been widely investigated [18-20], along with the possibilities to reach this regime with fewer electrons [21-23] and its nonadiabatic modulation to emit nonclassical states of light [24,25]. More in line with the present proposal, devices such as amplitude modulators are under development [26,27] and novel approaches to study transport in polaritonic systems are emerging [28,29].

In this Letter, we propose a new strategy to exploit absorption saturation of ISB transitions and trigger their nonlinear behavior with moderate pumping powers. The crucial aspect of this Letter is that we rely on the collapse of the light-matter coupling resulting from the saturation of the transition to produce a strong spectral feature. We show that the key to reduce the saturation intensity is to engineer the crossover between the weak (WCR) and the strong (SCR) coupling regimes so that it appears at a low doping. In contrast with the absorption saturation of interband excitations, both in the WCR and the SCR, we do not rely on Pauli blocking like traditional SESAM mirrors [2] nor on polariton-polariton interactions that dominate the nonlinear response of exciton polaritons $[4,5]$. We develop a unified analytical formalism for absorption saturation covering both the WCR and the SCR. We provide a set of simple analytical formulas that permit one to assess the saturation power levels for a given ISB system. Countering intuition, we show that, in the strong light-matter coupling regime, $I_{\text {sat }}$ increases linearly with the light-matter coupling strength (i.e., with the doping), while in the WCR it remains doping independent. To illustrate the importance of this finding, we present the design of a SESAM with extremely low saturation intensity and further derive the conditions to reach bistability. This formalism could apply to other systems hosting collective excitations in the strong coupling regime like vibrational absorbers with an adequate description of the population saturation of the excited state.

Let us start by an intuitive description of the saturation intensity in both coupling regimes. We model the saturation with a simple rate equation approach. The steady-state expression of the surface charge density $n_{2}$ in the excited subband of a system of doped QWs with surface charge density per QW $n_{s}$ is

$n_{2}=\frac{I}{\hbar \omega} \tau_{12} \mathcal{A}^{\mathrm{QW}}(\Delta n, \omega)=\frac{I}{N_{\mathrm{QW}} \hbar \omega} \tau_{12} \mathcal{A}^{\mathrm{ISB}}(\Delta n, \omega)$,

where $I$ is the incident intensity per unit surface, $\tau_{12}$ is the upper state lifetime, $N_{\mathrm{QW}}$ is the number of QWs, $\mathcal{A}^{\mathrm{QW}}(\Delta n, \omega)$ is the absorption per $\mathrm{QW}, \mathcal{A}^{\mathrm{ISB}}(\Delta n, \omega)$ is the total absorption of the ISB system, and $\Delta n=n_{1}-n_{2}$ is the population difference. The saturation condition is defined as $n_{2}=\left(n_{s} / 4\right) \Leftrightarrow \Delta n=\left(n_{s} / 2\right)$. The saturation intensity is thus derived from

$$
\frac{n_{s}}{4}=\frac{I_{\mathrm{sat}}}{N_{\mathrm{QW}} \hbar \omega} \tau_{12} \mathcal{A}^{\mathrm{ISB}}\left(\frac{n_{s}}{2}, \omega\right) .
$$

Remarkably, as long as the absorption from the ISB system can be written as a linear term in the doping density $n_{s}, I_{\text {sat }}$ is doping independent. While this is explicit in the bulk saturation intensity $I_{\text {sat }}^{0}$ from Eq. (2), this is, in fact, a general feature that also applies in the presence of a cavity when the system operates in the WCR. The absolute value $I_{\text {sat }}$ can be dramatically improved using a cavity, but it remains independent of the doping density because, in the WCR, the absorption from the ISB system is still a linear term in $n_{s}$. Instead, in the SCR, the absorption from the ISB system is independent of the doping because any further increase in the charge concentration results in an increase of the Rabi splitting (see Supplemental Material [30]). It is then straightforward to infer from Eq. (4) a saturation intensity that increases linearly with $n_{s}$ in SCR, in stark contrast with the WCR case.

To gain further insight into the physics at play, we use the temporal coupled mode theory (TCMT) formalism to derive a simple set of analytical expressions and unify both coupling regimes. In this framework, the cavity mode and the ISB system are modeled as oscillators with characteristic parameters $\left(\omega_{i}, \gamma_{i}, \Gamma_{i}\right)$ representing, respectively, their natural oscillation frequency and nonradiative and radiative dampings, as schematically depicted in Fig. 1(a). In the case of the ISB system, the radiative coupling of the ISB transition to free-space radiation is negligible [31,32]. The coupled system's response to an external excitation is given by

$$
\begin{gathered}
\frac{d a_{\mathrm{ISB}}}{d t}=\left(i \omega_{\mathrm{ISB}}-\gamma_{\mathrm{ISB}}\right) a_{\mathrm{ISB}}+i \Omega_{\mathrm{Rabi}} a_{c}, \\
\frac{d a_{c}}{d t}=\left(i \omega_{c}-\gamma_{\mathrm{nr}}-\Gamma_{r}\right) a_{c}+i \Omega_{\mathrm{Rabi}} a_{\mathrm{ISB}}+\sqrt{2 \Gamma_{r}} s^{+} \\
s^{-}=-s^{+}+\sqrt{2 \Gamma_{r}} a_{c}, \\
n_{2}=2 \tau_{12} \gamma_{\mathrm{ISB}} \frac{\left|a_{\mathrm{ISB}}\right|^{2}}{N_{\mathrm{QW}} \hbar \omega},
\end{gathered}
$$

where $a_{i}$ is the amplitude of oscillator $i$ referencing either the ISB system $(i=\mathrm{ISB})$ or the cavity $(i=c), \gamma_{\mathrm{ISB}}$ and $\gamma_{\mathrm{nr}}$ are the nonradiative decay rates, respectively, of the ISB transition and of the cavity, $\Gamma_{r}$ is the cavity radiative decay rate, and $s^{+}$and $s^{-}$are the amplitudes of the incoming and reflected fields. The coupling rate between the two oscillators is the vacuum Rabi frequency, which depends on the population difference

$$
\Omega_{\mathrm{Rabi}}^{2}=f_{w} \frac{\Delta n e^{2}}{4 \varepsilon \varepsilon_{0} m^{*} L_{\mathrm{QW}}}=a \Delta n,
$$


where $f_{w}$ is the filling fraction of the QWs material in the active region. Equations (8) and (9) ensure the selfconsistency of the set of TCMT equations. We assume an ISB linewidth independent of the doping density. While this is true for most of the values used throughout this Letter, at very large doping densities the ISB linewidth can increase [33]. Careful positioning of the dopants and growth interruption techniques can compensate for this increase [34]. We thus keep $\gamma_{\text {ISB }}$ constant. Our formalism applies to the case where only the lowest subband is occupied and only the fundamental transition plays a role. Solving this system in the harmonic regime $\left(s^{+}=\mathrm{e}^{i \omega t}\right)$, we derive the reflectivity of the coupled system $R=\left|s^{-} / s^{+}\right|^{2}$ (see Supplemental Material [30]) and the absorption of the sole ISB system,

$$
\mathcal{A}^{\mathrm{ISB}}=\frac{4 \gamma_{\mathrm{ISB}} \Gamma_{r} \Omega_{\mathrm{Rabi}}^{2}}{\left\{\gamma_{\mathrm{ISB}}\left(\gamma_{\mathrm{nr}}+\Gamma_{r}\right)-\left[\left(\omega-\omega_{0}\right)^{2}-\Omega_{\mathrm{Rabi}}^{2}\right\}^{2}+\left(\gamma_{\mathrm{ISB}}+\gamma_{\mathrm{nr}}+\Gamma_{r}\right)^{2}\left(\omega-\omega_{0}\right)^{2}\right.},
$$

assuming without loss of generality that the cavity and ISB system are resonant $\left(\omega_{\mathrm{ISB}}=\omega_{c}=\omega_{0}\right)$.

Using these equations, we study the response of the cavity-coupled ISB system as a function of the doping density $n_{s}$ for a weak probe beam. The maximum ISB absorption following Eq. (10) is shown in Fig. 1(b). Two regimes appear clearly: for low carrier density, the absorption increases linearly with $n_{s}$, while for large doping, the maximum ISB absorption flattens and becomes doping independent. The transition from the WCR to the SCR produces a clear spectral signature with the energy splitting of the two resonances [Fig. 1(b), inset]. The expression of (a)
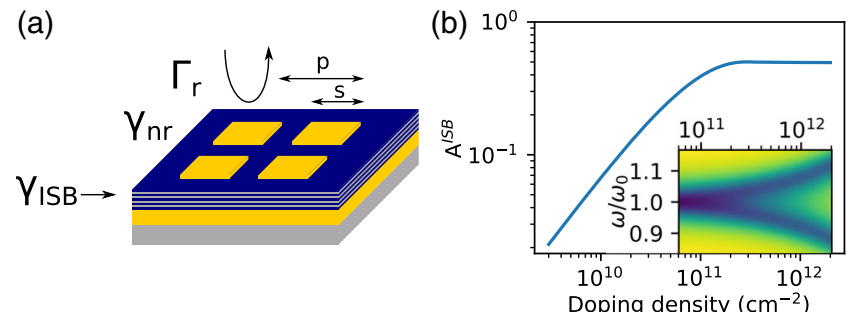

(c)

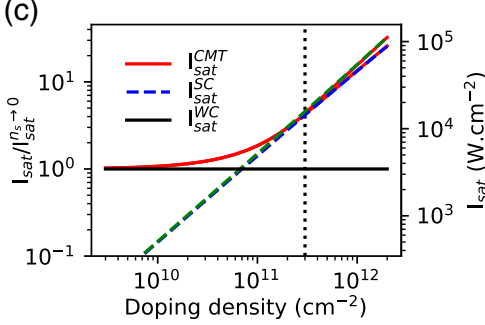

(d)

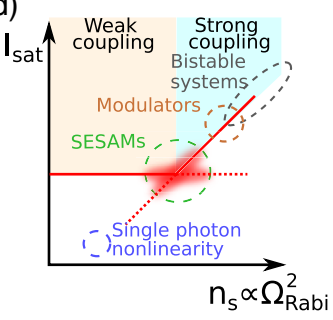

FIG. 1. (a) Sketch of the QWs embedded in a microcavity array with relevant parameters. (b) Intersubband absorption $\mathcal{A}^{\mathrm{ISB}}\left(n_{s}, \omega\right)$ as a function of $n_{s}$. Inset: reflectivity of the system as a function of $n_{s}$. (c) Saturation intensity normalized to $I_{\text {sat }}^{\mathrm{WC}}$ (left axis) or following our proposal (right axis) as a function of $n_{s}$. The black solid line is the asymptotic saturation intensity in the WCR $I_{\text {sat }}^{\mathrm{WC}}$, and the two dashed lines are the asymptotic saturation intensity $I_{\text {sat }}^{\mathrm{SC}}$ in the SCR corresponding to the lower (blue) and upper (green) polaritons. The red solid line is the analytical solution $I_{\text {sat }}^{ \pm, \text {CMT }}$. The black dotted line shows the proposed design for low saturation SESAMs. (d) Sketch of the two operating regimes and potential devices as a function of $n_{s}$. the ISB absorption (10) can be simplified in the two limiting cases of very low doping (WCR) or large doping (SCR). Plugging the asymptotic values of $\mathcal{A}^{\mathrm{ISB}}$ into Eq. (4) leads to analytical expressions for the saturation intensity,

$$
\begin{gathered}
I_{\mathrm{sat}}^{\mathrm{WC}}=\frac{\hbar \omega_{0} \varepsilon \varepsilon_{0} m^{*} L_{\mathrm{QW}} N_{\mathrm{QW}}}{2 \tau_{12} f_{w} e^{2}} \frac{\gamma_{\mathrm{ISB}}\left(\gamma_{\mathrm{nr}}+\Gamma_{r}\right)^{2}}{\Gamma_{r}}, \\
I_{\mathrm{sat}}^{\mathrm{SC}}=\frac{n_{s} \hbar \omega_{ \pm} N_{\mathrm{QW}}}{16 \tau_{12}} \frac{\left(\gamma_{\mathrm{ISB}}+\gamma_{\mathrm{nr}}+\Gamma_{r}\right)^{2}}{\gamma_{\mathrm{ISB}} \Gamma_{r}},
\end{gathered}
$$

where $\omega_{ \pm}$represents the polariton frequencies.

$I_{\text {sat }}^{\mathrm{WC}}$ [black solid line in Fig. 1(c)] represents the limit of low doping: the system operates in the weak coupling regime and $I_{\mathrm{sat}}^{\mathrm{WC}}$ is evidently independent of the doping density. Conversely, $I_{\text {sat }}^{\mathrm{SC}}$ [dashed lines in Fig. 1(c)] corresponds to a system operating in the strong coupling regime: the saturation intensity increases linearly with $n_{s}$ and $N_{\mathrm{QW}}$, confirming our previous intuitive discussion.

We rearrange the weak coupling limit expression (11) to highlight the key parameters governing the reduction in $I_{\text {sat }}$ once the QWs are placed in cavity. In line with the rest of this Letter, we assume a metal-insulator-metal (MIM) geometry, so that the electric field is almost constant across the active region,

$$
I_{\mathrm{sat}}^{\mathrm{WC}}=f_{\mathrm{osc}} \frac{\pi L_{\mathrm{AR}}}{\left(\lambda / n_{\mathrm{opt}}\right)} \frac{Q_{r}}{2\left(Q_{\mathrm{cav}}^{\mathrm{tot}}\right)^{2}} I_{\mathrm{sat}}^{0},
$$

where $f_{\text {osc }}$ is the ISB oscillator strength [6], $L_{\mathrm{AR}}$ is the total thickness of the active region, and $Q_{r}$ and $Q_{\mathrm{cav}}^{\text {tot }}$ are the cavity radiative and total $Q$ factor, respectively. Equation (13) explicitly links the lowest saturation intensity one can achieve in a cavity-coupled system $\left(I_{\text {sat }}^{\mathrm{WC}}\right)$ to the cavity-free one. Leaving aside the (fixed) oscillator strength, Eq. (13) shows that two physical effects lead to a reduction in $I_{\text {sat }}$. The first one stems from the compression of the electric field in ultrasubwavelength volumes. The second one is the branching ratio of the cavity quality factors. Its maximization requires both avoiding material 

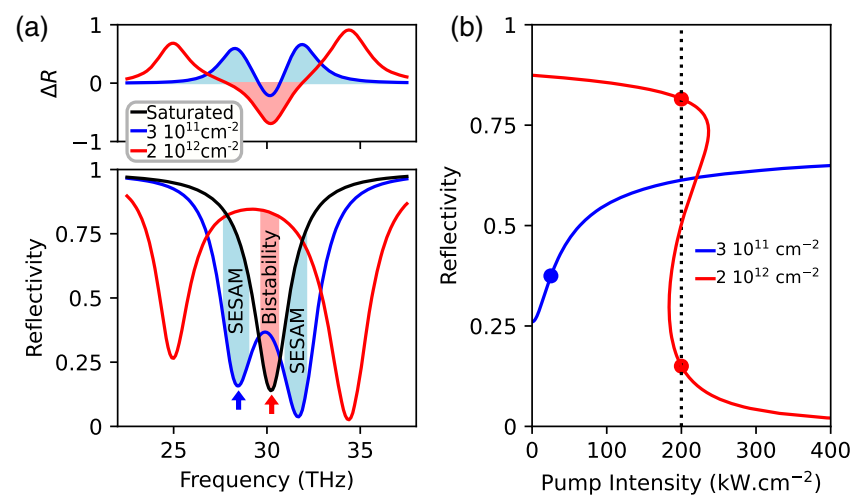

FIG. 2. (a) Bottom: FEM reflectivity simulations for two different doping densities. Blue, red: low incident power, the polaritonic states are present. Black: high incident power, only the cavity resonance is visible. The arrows indicate the pump frequency used in (b). (a) Top: differential reflectivity $\Delta R$. (b) Reflectivity of the two design samples, SESAM (blue, $n_{s}=310^{11} \mathrm{~cm}^{-2}$ ) and bistable (red, $n_{s}=210^{12} \mathrm{~cm}^{-2}$ ) as a function of pump intensity obtained from TCMT at the frequencies shown with arrows in (a).

losses (large $Q_{\mathrm{nr}}$ ) while retaining the critical coupling condition $Q_{r}=Q_{\mathrm{nr}}$ to ensure maximal energy feeding into the system.

The exact analytical solution is obtained by substituting Eq. (10) in Eq. (4) [red solid line in Fig. 1(c)], demonstrating the system's behavior around the crossing point and the broad span of ISB absorption saturation engineering that can be obtained with different doping densities. We summarize these different perspectives in Fig. 1(d). Ultrafast modulators relying on SCR [27], as well as harmonic generation with metasurfaces [26,35] suit the ultrastrong coupling regime, as they must not easily saturate. The regime of ultralow power nonlinearities in the mid-IR is an interesting perspective. While this requires very low loss resonators and is beyond the scope of this Letter, we speculate that mid-IR on chip III-V photonics is a suitable platform [36]. More interestingly, we anticipate the possibility to design and demonstrate SESAM mirrors. Such a device would best fit the onset of SCR, as low $I_{\text {sat }}$ can be achieved, retaining at the same time a high reflectivity in the saturated state.

We rely on this formalism to devise a semiconductorbased saturable system operating with low $I_{\text {sat }}$ at mid-IR wavelengths $\left(\omega_{0}=30 \mathrm{THz}, \lambda=10 \mu \mathrm{m}\right)$. A superficial inspection of Fig. 1(c) suggests that the lower the doping, the better. However, in the case where the system operates in the weak coupling regime, the main practical limitation arises from the overlap factor $f_{w}$ [see Eq. (11)] that must remain elevated. In the mid-IR, overlap factors around unity are obtained only using MIM cavities, while dielectric cavities result in very low $f_{w}$ values. In turn, saturation would be almost undetectable in a MIM cavity operating in the WCR, since the losses are dominated by the cavity, and the saturated and nonsaturated reflectivity spectra would be almost identical. It is then necessary to operate in the SCR, where saturation leads to clear spectral changes. Equation (12) clarifies that the system must operate at the onset of strong coupling, embed the lowest possible number of QWs, and the lowest possible sheet doping per QW, allowing operation in the SCR. We consider a system of four periods of $\mathrm{GaAs} / \mathrm{Al}_{0.33} \mathrm{Ga}_{0.67} \mathrm{As}$ $(8.3 / 14 \mathrm{~nm})$, with barriers $\delta$ doped to $n_{s}=3 \times 10^{11} \mathrm{~cm}^{-2}$, embedded in metal-metal patch-cavity resonators [Fig. 1(a)] [18,35,37-39]. The structure thickness is $\approx 140 \mathrm{~nm}$, and the overlap factor is $f_{w} \approx 0.24$.

We numerically calculate the system reflectivity in strong coupling with a patch-cavity mode at low incident power using finite element method (FEM) simulations [Fig. 2(a) blue curve, lower panel]: the upper polariton and lower polariton modes are clearly visible. The black curve shows instead the saturated system $\left(I>I_{\text {sat }}\right)$. The top panel shows the differential reflectivity. The regions highlighted in light blue correspond to an absorption that decreases with incident power: they suit the development of SESAM mirrors. The regions highlighted in red correspond to an absorption that increases with incident power: we will discuss later how, under specific conditions, a bistable system could be implemented here.

From the FEM reflectivity spectra, we can extract the relevant system parameters to use as inputs into the TCMT, respectively, $\gamma_{\mathrm{nr}}=0.02 \omega_{c}$ and $\Gamma_{r}=0.02 \omega_{c}$. The nonradiative damping rate of the ISB system is set with typical intersubband absorption $Q$ factors, $\gamma_{\mathrm{ISB}}=0.05 \omega_{\mathrm{ISB}}$. We calculate $I_{\text {sat }}$ as a function of the doping for these sample parameters, as reported in Fig. 1(c). We obtain a WCR limit value of $I_{\mathrm{sat}}^{\mathrm{WC}}=3.45 \mathrm{~kW} / \mathrm{cm}^{2}$. Considering the previously chosen doping level of $3 \times 10^{11} \mathrm{~cm}^{-2}$ per QW, we get $I_{\text {sat }}^{\mathrm{SC}}=14.7 \mathrm{~kW} / \mathrm{cm}^{2}$, with a Rabi frequency $\Omega_{\mathrm{Rabi}} \approx 3 \mathrm{THz}$. Note that the saturation intensity of the same active region in absence of a cavity is around $I_{\text {sat }}^{0}=1.1 \mathrm{MW} \mathrm{cm}^{-2}$. Our approach allows at least an 80 -fold reduction of the saturation intensity.

This result, which already represents a record-low value for mid-IR absorption saturation in an ISB system, is an upper limit. Arranging microcavities in an array enhances their absorption cross section as they can collect photons over an area greater than their physical area [35,40,41]. Numerically optimizing the array absorption $(s=1.2$, $p=2.2 \mu \mathrm{m}$ ), the active surface covers $30 \%$ of the unit cell. Hence, we estimate a corrected saturation intensity $I_{\text {sat }}^{\text {enh }}=0.3 \times I_{\text {sat }}^{\text {SC }} \approx 4.4 \mathrm{~kW} / \mathrm{cm}^{2}$.

So far, we have only focused on quantifying and engineering $I_{\text {sat }}$. Equations (5)-(9) also allow one to derive the steady-state response of the system to a variable pump power. This is particularly useful to predict experimental data on the observation of nonlinear optical properties of the coupled system. We follow the method developed for exciton polaritons in Ref. [5] and express the reflected 
beam intensity from the population of the excited state using the TCMT equations. It is best demonstrated by writing the intensity-dependent excited-state population as a function of the coupled system's parameters. For simplicity we assume without loss of generality that the pump frequency is set to $\omega=\omega_{0}$,

$I\left(n_{2}\right)=\frac{n_{2} \hbar \omega_{0} N_{\mathrm{QW}}\left\{2 a n_{2}-\left[\gamma_{\mathrm{ISB}}\left(\gamma_{\mathrm{nr}}+\Gamma_{r}\right)-a n_{s}\right]\right\}^{2}}{4 \tau_{12} \gamma_{\mathrm{ISB}} \Gamma_{r} a\left(n_{s}-2 n_{2}\right)}$.

The derivation and its extension to the detuned pump $\left(\omega \neq \omega_{0}\right)$ used to describe the SESAM operation are presented in Supplemental Material [30]. Under certain conditions, Eq. (14) admits several solutions: multiple values of $n_{2}$ can satisfy the equation for a given pump intensity $I$, the system is then said to exhibit "optical bistability." The emergence of bistability in ISB systems has been previously theoretically approached in Refs. $[15,42,43]$, either through charge transfer or absorption saturation.

The observation of bistability in the system requires very large changes in absorption and is thus usually predicted at prohibitively large pumping intensities [42]. We show here that it can emerge at lower pumping powers. From Eq. (14), the minimal doping necessary to observe bistability as a function of the system's damping rates is

$$
n_{s}>\frac{32 \varepsilon \varepsilon_{0} m^{*} L_{\mathrm{QW}} \gamma_{\mathrm{ISB}}\left(\gamma_{\mathrm{nr}}+\Gamma_{r}\right)}{f_{w} e^{2}} .
$$

Using the previous parameters, this critical value is around $n_{s} \geq 1.1 \times 10^{12} \mathrm{~cm}^{-2}$. To illustrate this regime of operation, we explore a sample with a doping level $n_{s}=2 \times 10^{12} \mathrm{~cm}^{-2}$. FEM calculations of the bistable sample are presented in Fig. 2(a) (red lines). Figure 2(d) shows the reflected beam intensity when pumping the SESAM device at $\omega_{-} \approx 28 \mathrm{THz}$ and the bistable device at $\omega_{0}=30 \mathrm{THz}$. We evidence a bistable behavior for pump intensities around $200 \mathrm{~kW} / \mathrm{cm}^{2}$ (red curve) with a classical $\mathrm{S}$-shape reflectivity curve. The saturable absorber sample with a doping level $n_{s}=3 \times 10^{11} \mathrm{~cm}^{-2}$ pumped at the lower polariton frequency (blue) shows a classical saturation behavior, with $I_{\text {sat }}$ marked as the blue circle.

Remembering the definition of plasma frequency [44], Eq. (15) can be recast in a universal form, linking it to modern cavity (quantum) electrodynamics concepts,

$$
\frac{\Omega_{\mathrm{Rabi}}}{\omega_{\mathrm{ISB}}} \times \frac{\Omega_{\mathrm{Rabi}}}{\omega_{\mathrm{cav}}}>\frac{2}{Q_{\mathrm{ISB}} Q_{\mathrm{cav}}^{\mathrm{tot}}} \Leftrightarrow C>8,
$$

where $C=\Omega_{\mathrm{Rabi}}^{2} / \gamma_{\mathrm{ISB}}\left(\gamma_{\mathrm{nr}}+\Gamma_{r}\right)$ is the cooperativity. Remarkably, this result is extremely similar to the bistability criterion reported for atomic ensembles in interferometers and cavities [45,46]. Equation (16) explicitly spells out the physical parameters governing bistability in an ISB polariton system, which has never been experimentally reported.

In conclusion, we have demonstrated that the nature of absorption saturation for an ISB system changes radically when the system transitions from the weak to the strong light-matter coupling regime. In particular, in the SCR, the saturation intensity becomes doping dependent. This brings a new degree of freedom and reveals that the true nature of absorption saturation emerges when we encompass the full range of possible light-matter couplings, from the weak up to the strong coupling regime. Our Letter shows the richness of possibilities to tailor ISB absorption in strong coupling with respect to the weak coupling case. We have further provided design rules to enable record-low threshold intensity saturable and bistable mid-IR systems.

We thank I. Carusotto for thorough reading of the Letter and F. H. Julien, M. Helm, L. Andreani, A. Bousseksou, and S. Pirotta, for discussions. We acknowledge financial support from the European Union FET-Open Grant MIRBOSE (No. 737017) and from the French National Research Agency (project TERASEL, ANR 18-CE240013 and project SOLID, ANR 19-CE24-0003).

Note added.-Recently, we came aware of new work showing ISB polariton saturation in metasurfaces [47]. Our theory quantitatively reproduces the results of Ref. [47].

*raffaele.colombelli@u-psud.fr

[1] R. W. Boyd, Nonlinear Optics, 3rd ed. (Elsevier, Amsterdam, 2008).

[2] U. Keller, D. A. B. Miller, G. D. Boyd, T. H. Chiu, J. F. Ferguson, and M. T. Asom, Solid-state low-loss intracavity saturable absorber for ND:YLF lasers: An antiresonant semiconductor Fabry-Perot saturable absorber, Opt. Lett. 17, 505 (1992).

[3] B. Willenberg, J. Maurer, B. W. Mayer, and U. Keller, Subcycle time resolution of multi-photon momentum transfer in strong-field ionization, Nat. Commun. 10, 5548 (2019).

[4] A. Tredicucci, Y. Chen, V. Pellegrini, M. Börger, and F. Bassani, Optical bistability of semiconductor microcavities in the strong-coupling regime, Phys. Rev. A 54, 3493 (1996).

[5] A. Baas, J. P. Karr, H. Eleuch, and E. Giacobino, Optical bistability in semiconductor microcavities, Phys. Rev. A 69, 023809 (2004).

[6] M. Helm, The basic physics of intersubband transitions, in Semiconductors and Semimetals edited by H. Liu and F. Capasso (Elsevier, New York, 1999), Vol. 62, Chap. 1, pp. 1-99.

[7] A. Baranov and E. Tournie, Semiconductor Lasers: Fundamentals and Applications (Woodhead Publishing, Cambridge, 2013). 
[8] E. Rosencher, B. Vinter, P. Piva, and K. Firm, Optoelectronics (Cambridge University Press, Cambridge, England, 2002).

[9] A. Seilmeier, H.-J. Hübner, G. Abstreiter, G. Weimann, and W. Schlapp, Intersubband Relaxation in GaAs-al $\mathrm{ga}_{1-x}$ as Quantum Well Structures Observed Directly by an Infrared Bleaching Technique, Phys. Rev. Lett. 59, 1345 (1987).

[10] F. H. Julien, J. Lourtioz, N. Herschkorn, D. Delacourt, J. P. Pocholle, M. Papuchon, R. Planel, and G. Le Roux, Optical saturation of intersubband absorption in GaAs- $\mathrm{Al}_{x} \mathrm{Ga}_{1-x} \mathrm{As}$ quantum wells, Appl. Phys. Lett. 53, 116 (1988).

[11] K. L. Vodopyanov, V. Chazapis, C. C. Phillips, B. Sung, and J. S. Harris, Intersubband absorption saturation study of narrow III-V multiple quantum wells in the $\lambda=2.8-9 \mu \mathrm{m}$ spectral range, Semicond. Sci. Technol. 12, 708 (1997).

[12] D. Dini, R. Köhler, A. Tredicucci, G. Biasiol, and L. Sorba, Microcavity Polariton Splitting of Intersubband Transitions, Phys. Rev. Lett. 90, 116401 (2003).

[13] S. De Liberato and C. Ciuti, Stimulated Scattering and Lasing of Intersubband Cavity Polaritons, Phys. Rev. Lett. 102, 136403 (2009).

[14] A. Delteil, A. Vasanelli, P. Jouy, D. Barate, J. C. Moreno, R. Teissier, A. N. Baranov, and C. Sirtori, Optical phonon scattering of cavity polaritons in an electroluminescent device, Phys. Rev. B 83, 081404(R) (2011).

[15] S. Zanotto, F. Bianco, L. Sorba, G. Biasiol, and A. Tredicucci, Saturation and bistability of defect-mode intersubband polaritons, Phys. Rev. B 91, 085308 (2015).

[16] R. Colombelli and J.-M. Manceau, Perspectives for Intersubband Polariton Lasers, Phys. Rev. X 5, 011031 (2015).

[17] J.-M. Manceau, N.-L. Tran, G. Biasiol, T. Laurent, I. Sagnes, G. Beaudoin, S. De Liberato, I. Carusotto, and R. Colombelli, Resonant intersubband polariton-LO phonon scattering in an optically pumped polaritonic device, Appl. Phys. Lett. 112, 191106 (2018).

[18] Y. Todorov, A. M. Andrews, R. Colombelli, S. De Liberato, C. Ciuti, P. Klang, G. Strasser, and C. Sirtori, Ultrastrong Light-Matter Coupling Regime with Polariton Dots, Phys. Rev. Lett. 105, 196402 (2010).

[19] G. Scalari, C. Maissen, D. Turcinkova, D. Hagenmuller, S. De Liberato, C. Ciuti, C. Reichl, D. Schuh, W. Wegscheider, M. Beck, and J. Faist, Ultrastrong coupling of the cyclotron transition of a 2D electron gas to a $\mathrm{THz}$ metamaterial, Science 335, 1323 (2012).

[20] A. Delteil, A. Vasanelli, Y. Todorov, C. Feuillet Palma, M. Renaudat St-Jean, G. Beaudoin, I. Sagnes, and C. Sirtori, Charge-Induced Coherence between Intersubband Plasmons in a Quantum Structure, Phys. Rev. Lett. 109, 246808 (2012).

[21] M. Malerba, T. Ongarello, B. Paulillo, J.-M. Manceau, G. Beaudoin, I. Sagnes, F. De Angelis, and R. Colombelli, Towards strong light-matter coupling at the single-resonator level with sub-wavelength mid-infrared nano-antennas, Appl. Phys. Lett. 109, 021111 (2016).

[22] J. Keller, G. Scalari, S. Cibella, C. Maissen, F. Appugliese, E. Giovine, R. Leoni, M. Beck, and J. Faist, Few-electron ultrastrong light-matter coupling at $300 \mathrm{GHz}$ with nanogap hybrid LC microcavities, Nano Lett. 17, 7410 (2017).

[23] M. Jeannin, G. Mariotti Nesurini, S. Suffit, D. Gacemi, A. Vasanelli, L. H. Li, A. G. Davies, E. H. Linfield, C. Sirtori, and Y. Todorov, Ultra-strong light-matter coupling in deeply subwavelength THz LC resonators, ACS Photonics 6, 1207 (2019).

[24] C. Ciuti, G. Bastard, and I. Carusotto, Quantum vacuum properties of the intersubband cavity polariton field, Phys. Rev. B 72, 115303 (2005).

[25] G. Günter, A. A. Anappara, J. Hees, A. Sell, G. Biasiol, L. Sorba, S. De Liberato, C. Ciuti, A. Tredicucci, A. Leitenstorfer, and R. Huber, Sub-cycle switch-on of ultrastrong light-matter interaction, Nature (London) 458, 178 (2009).

[26] J. Lee, S. Jung, P.-Y. Chen, F. Lu, F. Demmerle, G. Boehm, M.-C. Amann, A. AlÃ, and M. A. Belkin, Ultrafast electrically tunable polaritonic metasurfaces, Adv. Opt. Mater. 2, 1057 (2014).

[27] S. Pirotta, N.-L. Tran, G. Biasiol, A. Jollivet, P. Crozat, J.-M. Manceau, A. Bousseksou, and R. Colombelli, Fast amplitude modulation up to $1.5 \mathrm{GHz}$ of mid-IR free-space beams at room-temperature, Nat. Commun. 12, 799 (2021).

[28] P.-B. Vigneron, S. Pirotta, I. Carusotto, N.-L. Tran, G. Biasiol, J.-M. Manceau, A. Bousseksou, and R. Colombelli, Quantum well infrared photo-detectors operating in the strong light-matter coupling regime, Appl. Phys. Lett. 114, 131104 (2019).

[29] B. Limbacher, M. A. Kainz, S. Schoenhuber, M. Wenclawiak, C. Derntl, A. M. Andrews, H. Detz, G. Strasser, A. Schwaighofer, B. Lendl, J. Darmo, and K. Unterrainer, Resonant tunneling diodes strongly coupled to the cavity field, Appl. Phys. Lett. 116, 221101 (2020).

[30] See Supplemental Material at http://link.aps.org/supplemental/ 10.1103/PhysRevLett.127.187401 for details on: temporal coupled mode theory; intersubband absorption and saturation intensities; bistability.

[31] F. Alpeggiani and L. C. Andreani, Semiclassical theory of multisubband plasmons: Nonlocal electrodynamics and radiative effects, Phys. Rev. B 90, 115311 (2014).

[32] M. Jeannin, T. Bonazzi, D. Gacemi, A. Vasanelli, L. Li, A. G. Davies, E. Linfield, C. Sirtori, and Y. Todorov, Absorption engineering in an ultrasubwavelength quantum system, Nano Lett. 20, 4430 (2020).

[33] R. J. Warburton, K. Weilhammer, J.P. Kotthaus, M. Thomas, and H. Kroemer, Influence of Collective Effects on the Linewidth of Intersubband Resonance, Phys. Rev. Lett. 80, 2185 (1998).

[34] N. L. Tran, G. Biasiol, A. Jollivet, A. Bertocci, F. H. Julien, J.-M. Manceau, and R. Colombelli, Evidence of intersubband linewidth narrowing using growth interruption technique, Photonics 6, 38 (2019).

[35] J. Lee, N. Nookala, J. S. Gomez-Diaz, M. Tymchenko, F. Demmerle, G. Boehm, M.-C. Amann, A. Al ̃̃, and M. A. Belkin, Ultrathin second-harmonic metasurfaces with record-high nonlinear optical response, Adv. Opt. Mater. 4, 664 (2016).

[36] I. Roland, A. Borne, M. Ravaro, R. D. Oliveira, S. Suffit, P. Filloux, A. Lemaître, I. Favero, and G. Leo, Frequency doubling and parametric fluorescence in a four-port aluminum gallium arsenide photonic chip, Opt. Lett. 45, 2878 (2020).

[37] C. A. Balanis, Antenna Theory: Analysis and Design (Wiley-Interscience, New Jersey, 2005). 
[38] M. Hakl, Q. Lin, S. Lepillet, M. Billet, J.-F. Lampin, S. Pirotta, R. Colombelli, W. Wan, J. C. Cao, H. Li, E. Peytavit, and S. Barbieri, Ultrafast quantum-well photodetectors operating at $10 \mu \mathrm{m}$ with a flat frequency response up to $70 \mathrm{GHz}$ at room temperature, ACS Photonics 8, 464 (2021).

[39] E. Cortese, N.-L. Tran, J.-M. Manceau, A. Bousseksou, I. Carusotto, G. Biasiol, R. Colombelli, and S. De Liberato, Excitons bound by photon exchange, Nat. Phys. 17, 31 (2021).

[40] T. J. Seok, A. Jamshidi, M. Kim, S. Dhuey, A. Lakhani, H. Choo, P. J. Schuck, S. Cabrini, A. M. Schwartzberg, J. Bokor, E. Yablonovitch, and M. C. Wu, Radiation engineering of optical antennas for maximum field enhancement, Nano Lett. 11, 2606 (2011).

[41] C. Feuillet-Palma, Y. Todorov, A. Vasanelli, and C. Sirtori, Strong near field enhancement in $\mathrm{THz}$ nano-antenna arrays, Sci. Rep. 3, 1361 (2013).
[42] M. Seto and M. Helm, Charge transfer induced optical bistability in an asymmetric quantum well structure, Appl. Phys. Lett. 60, 859 (1992).

[43] M. Zaluzny, Saturation of intersubband absorption and optical rectification in asymmetric quantum wells, J. Appl. Phys. 74, 4716 (1993).

[44] M. Zaluzny and C. Nalewajko, Coupling of infrared radiation to intersubband transitions in multiple quantum wells: The effective-medium approach, Phys. Rev. B 59, 13043 (1999).

[45] P. Drummond, Optical bistability in a radially varying mode, IEEE J. Quantum Electron. 17, 301 (1981).

[46] D. E. Grant and H. J. Kimble, Optical bistability for two-level atoms in a standing-wave cavity, Opt. Lett. 7, 353 (1982).

[47] S. A. Mann, N. Nookala, S. C. Johnson, M. Cotrufo, A. Mekawy, J. F. Klem, I. Brener, M. B. Raschke, A. Alù, and M. A. Belkin, Ultrafast optical switching and power limiting in intersubband polaritonic metasurfaces, Optica 8, 606 (2021). 